\title{
Traditional and cumulative meta-analysis: Chemoradiotherapy followed by surgery versus surgery alone for resectable esophageal carcinoma
}

\author{
HAIMING FENG ${ }^{1 *}$, YE ZHAO $^{2 *}$, TAO JING $^{1}$, JIANXING MA $^{1}$, \\ YINGLU ZHAO ${ }^{3}$, JIANHUA ZHANG ${ }^{4}, \mathrm{CHENG} \mathrm{WANG}^{1 *}$ and $\mathrm{BIN} \mathrm{LI}^{1 *}$ \\ ${ }^{1}$ Department of Thoracic Surgery, The Second Affiliated Hospital of Lanzhou University; ${ }^{2}$ Evidence Based Medicine Center \\ of Lanzhou University; ${ }^{3}$ Department of Cardiac Surgery, The Second Affiliated Hospital of Lanzhou University, \\ Lanzhou, Gansu 730000; ${ }^{4}$ Department of Thoracic Surgery, Shenzhen Hospital \\ of Southern Medical University, Shenzhen, Guangdong 518100, P.R. China
}

Received March 23, 2017; Accepted November 17, 2017

DOI: $10.3892 / \mathrm{mco} .2017 .1534$

\begin{abstract}
The role of neoadjuvant chemoradiotherapy followed by surgery (CRTS) compared with surgery alone (SA) for resectable esophageal carcinoma has been established by several randomized controlled trials (RCTs). The present study aimed to investigate the difference in survival between the two treatments by a review of meta-analyses. Related research indicators were extracted from RCTs investigating CRTS or SA for resectable esophageal carcinoma by searching electronic databases for eligible articles. Outcomes were synthesized by adopting a fixed- or random-effects model with $95 \%$ confidence interval (CI). A total of 22 RCTs including 3,419 patients were selected. The odds ratio (OR) (95\% CI, P-value), expressed as CRTS vs. SA, was $1.06(0.94-1.19, \mathrm{P}=0.348)$ for 1 -year overall survival rate (OSR1y), 1.38 (1.20-1.58, $\mathrm{P}<0.001)$ for 3-year overall survival rate (OSR3y), and $1.42(1.22-1.66, \mathrm{P}<0.001)$ for 5-year overall survival rate (OSR5y). The R0 resection rate increased in patients treated by CRTS (OR=2.76, 95\% CI: 2.15-3.53, $\mathrm{P}<0.001)$. CRTS lowered the locoregional cancer recurrence $(\mathrm{OR}=0.49,95 \% \mathrm{CI}: 0.36-6.65, \mathrm{P}<0.001)$ and distant metastasis rate $(\mathrm{OR}=0.76,95 \% \mathrm{CI}: 0.60-0.97, \mathrm{P}=0.02)$. However, the incidence of postoperative mortality was similar between the two groups (OR=0.97, 95\% CI: 0.72-1.32,
\end{abstract}

Correspondence to: Dr Cheng Wang or Dr Bin Li, Department of Thoracic Surgery, The Second Affiliated Hospital of Lanzhou University, 82 Cuiyingmen, Chengguan, Lanzhou, Gansu 730000, P.R. China

E-mail: wangchengxw@qliyun.com

E-mail: drleebin@outlook.com

*Contributed equally

Key words: esophageal carcinoma, chemoradiotherapy, surgery, neoadjuvant therapy, meta-analysis
$\mathrm{P}=0.87$ ). The subgroup analysis revealed that OSR $3 y$ and OSR5y for Asian, European and American populations were significantly higher in the CRTS group compared with those in the SA group $(\mathrm{P}<0.05)$. When comparing the OSR1y between the two groups for patients in all three continents, there was no significant difference $(\mathrm{P}>0.05)$. Histological subgroup analysis indicated that patients with esophageal adenocarcinoma may benefit from CRTS in terms of OSR1y $(\mathrm{OR}=1.55,95 \% \mathrm{CI}: 1.09-2.20, \mathrm{P}=0.01)$, OSR3y $(\mathrm{OR}=1.77$, 95\% CI: $1.34-2.36, \mathrm{P}<0.0001)$ and OSR5y $(\mathrm{OR}=1.92,95 \% \mathrm{CI}$ : $1.34-2.75, \mathrm{P}=0.0004)$. The pooled $\mathrm{OR}$ of squamous cell carcinoma in terms of OSR3y and OSR5y between the two groups was 1.57 (95\% CI: 1.21-2.04, $\mathrm{P}=0.0006)$ and 1.69 (95\% CI: 1.32-2.16, $\mathrm{P}<0.0001)$, respectively, but there was no statistical difference in terms of OSR1y (OR=1.13, 95\% CI: 0.88-1.45, $\mathrm{P}=0.35$ ). Thus, neoadjuvant CRT followed by surgery may improve long-term survival and surgical parameters, and reduce locoregional cancer recurrence and distant metastasis.

\section{Introduction}

Esophageal cancer is the eighth most common type of cancer worldwide, with $>480,000$ new cases diagnosed annually (1). Esophageal cancer has a high mortality rate (sixth worldwide), causing $>400,000$ deaths annually (2). Squamous cell carcinoma is the most frequently type occurring in Asians, particularly in China, where it accounts for $70 \%$ of global morbidity (3). However, the incidence of esophageal adenocarcinoma in Western populations is rapidly increasing, whereas that of squamous cell carcinoma remains unchanged (4). Esophagectomy is considered to be the standard treatment for patients with resectable esophageal carcinoma, despite a detailed assessment of preoperative staging showing that $25 \%$ of patients treated with definitive surgery had microscopically positive resection margins (R1). However, the 5-year survival rate scarcely exceeds $40 \%$ (5); in addition, due to the morbidity and mortality associated with surgery, this approach is limited to a minority of medically fit patients. 
Since the 1980s, there have been several randomized clinical trials (RCTs) assessing the efficacy of preoperative chemoradiotherapy followed by surgery (CRTS) in the treatment of esophageal cancer. However, the sample-size of these RCTs was small, with a short-term follow-up and adverse outcomes in the surgical monotherapy arm of combination treatment trials when compared with surgery alone (SA) case-series (6). Furthermore, the majority of the trials did not have sufficient statistical power to produce a definitive conclusion. Thus, a comprehensive analysis was conducted to compare the potential objective value of CRTS with SA for resectable esophageal carcinoma.

As regards the differences between traditional and cumulative meta-analysis, cumulative meta-analysis refers to a meta-analysis of the obtained studies in a certain order; those studies are treated as a continuous whole and multiple meta-analyzes are performed by accumulating studies sequentially in a specified sequence (such as publication time). In addition, if a new test result is published, a new meta-analysis may follow. Traditional meta-analysis is performed only once, whereas cumulative meta-analysis is performed several times; the former may obtain summary results, but cannot distinguish the impact of each study result on the summary results, whereas the latter does not only obtain the results of the summary and compare the dynamic results of summary changes, but also compares the effect of the newly added studies on overall outcome. The cumulative meta-analysis is controversial in terms of test level. Some scholars object to performing multiple meta-analyses due to the increasing probability of committing class I error, and claim the test level should be adjusted for each analysis; some scholars believe that the analysis of the Bayesian theory may be used to explain, without the need for adjustment.

Based on this theory, the present study aimed to combine the traditional and cumulative meta-analysis to explore the pooled results of the relevant studies.

\section{Data collection methods}

Search strategy. The relevant articles identified were RCTs retrieved from Embase, PubMed and The Cochrane Library (issue 4,2016 ) and the deadline for trial publication and/or presentation was October 1st, 2016. The American Society of Clinical Oncology (ASCO) and the Cochrane Collaboration's Central Register of Controlled Clinical Trials were searched for updates of the trials. The search terms were as follows: Esophageal neoplasms, esophageal cancer, esophageal carcinoma, esophageal tumor, neoadjuvant therapy, chemoradiotherapy, esophagectomy, resection, surgery and operation.

Inclusion and exclusion criteria. In our meta-analysis, the study focus was locoregional resectable esophageal cancer patients who received either CRTS or SA. The eligible studies were required to meet the following inclusion criteria: i) Prospective RCTs comparing CRTS vs. SA in the initial management of resectable esophageal cancer; ii) outcome indices containing survival data; iii) no significant differences in baseline characteristics between the CRTS and SA groups; and iv) definitive follow-up survival number of cases or survival curve, with a follow-up rate of $>95 \%$ in the original RCTs. Studies focusing on patients with esophageal cancer who had been treated with neoadjuvant chemotherapy alone or radiotherapy alone, other studies without usable data, letters, editorials, case reports and reviews were excluded.

Data extraction and quality assessment. Two investigators independently extracted data to avoid bias in the course of the extraction. Disagreements were resolved by consensus or consultation with third parties. Statistics for each available outcome were extracted from trials in the light of the key information including patient characteristics, first author, year of publication, country/region, the regimen of the CRTS, and tumor histology. The methodological quality assessment of individual studies followed the Cochrane risk of bias method.

Statistical analysis. Overall survival rates at 1, 3 and 5 years (OSR1y, OSR3y and OSR5y, respectively), R0 resection rate, postoperative mortality, postoperative local recurrence rate and postoperative distant metastasis rate were extracted and pooled with $95 \%$ confidence intervals (CIs) by adopting the fixed- or random-effects model where heterogeneity was assessed with the inconsistency statistic $\left(\mathrm{I}^{2}<50 \%, \mathrm{P}>0.05\right.$; and $\mathrm{I}^{2} \geq 50 \%$, $\mathrm{P} \leq 0.05$, respectively). The odds ratio (OR) was estimated with 95\% CI and P-values in both the CRTS and SA groups. All calculations were performed using Review Manager 5.3 (Nordic Cochrane Centre, Copenhagen, Denmark), R software version 3.2.2, and STATA version 12.0 (StataCorp LP- College Station, TX, USA). Statistical significance was set at $\mathrm{P}<0.05$.

\section{Results}

Summary of included studies. A total of 1279 records were identified according to the search strategy and 22 were finally included in the meta-analysis after removing duplicated, ineligible and unrelated studies (Fig. 1). Ten countries, including China, Australia, Japan, Korea, Thailand, United States of America, France, The Netherlands, Ireland and Norway, were included in the RCTs. Of the 22 studies, 20 (7-26) reported OSR1y, 19 (7-21,24-27) reported OSR3y and $15(8,11-13,15-17,19-12,24-27)$ reported information on OSR5y after SA or CRTS for resectable esophageal carcinoma.

As regards pathological type, 5 RCTs $(10,15,24,26,27)$ on adenocarcinoma and 12 studies $(7-9,11,13,14,16,21,23,24,26)$ on squamous cell carcinoma investigated OSR1y, OSR 3y and OSR 5y after CRTS or SA for resectable esophageal carcinoma.

A total of 9 studies $(7,9,11,13-15,23,27,28)$ reported R0 resection rate, $10(9,12-15,16,19-21,26)$ included postoperative local recurrence rate and distant metastasis rate, and $15(7-12,14-16,20,21,28)$ provided postoperative mortality information.

With respect to the treatment efficacy of both methods in different countries or regions, 8 trials $(8,13,14,16-19,23)$ collected data from Asian populations, 7 studies (7-11,24-26) from European populations, and $2(12,20)$ from USA populations. The study characteristics are summarized in Table I.

Survival rate. The heterogeneity test at all the time points had a $\mathrm{I}^{2}$ value of $<55 \%$; thus, the fixed-effects model was used.

OSR1y, OSR 3y and OSR5y outcomes of traditional and cumulative meta-analysis. Traditional meta-analysis provided 


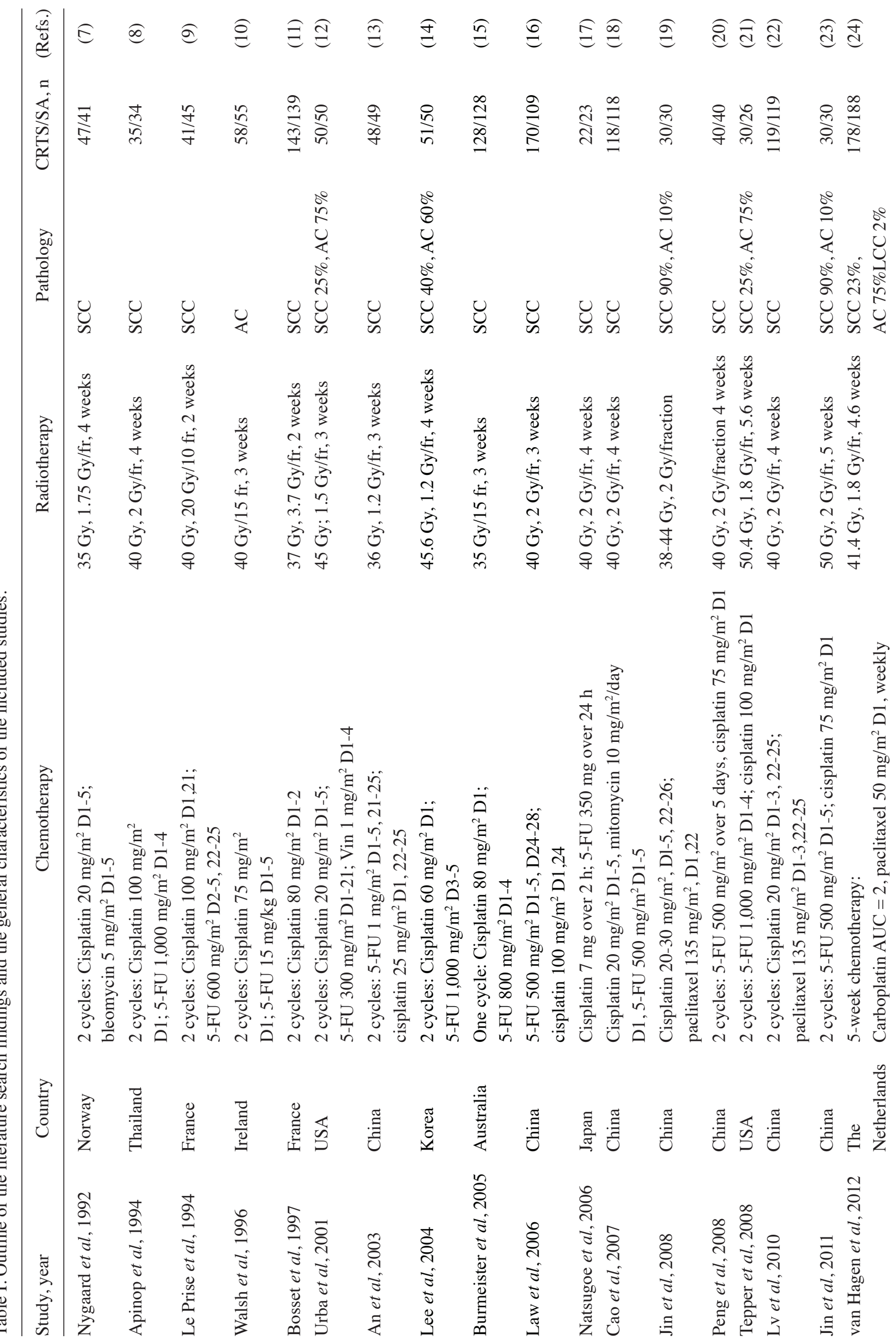




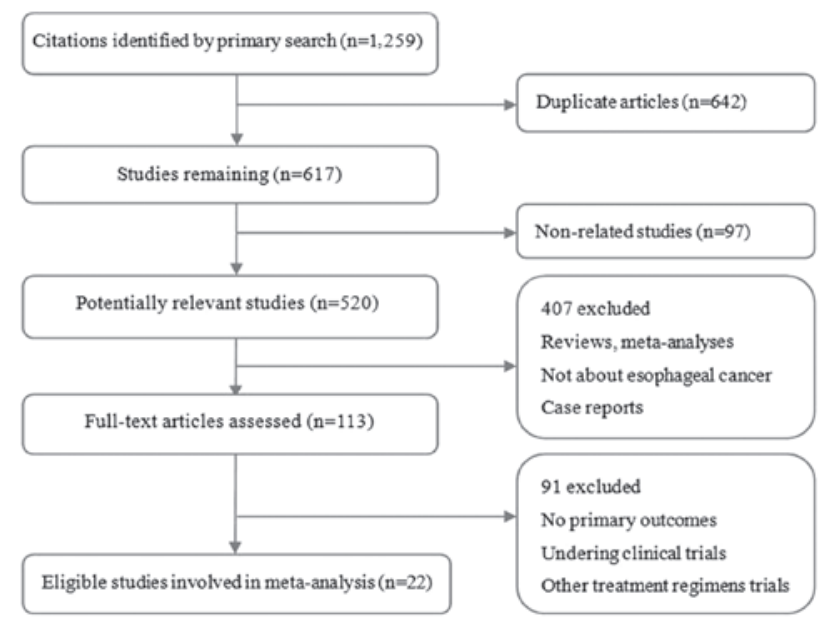

Figure 1. Trial selection process.

evidence that, compared with the SA group, the OSR3y and OSR5y were significantly higher in the CRTS group. The pooled OSR $3 y$ was $44 \%$ (95\% CI: $37-52 \%$ ) vs. $30 \%$ (95\% CI: $23-38 \%$ ), respectively, and the OSR5y was $36 \%$ (95\% CI: $32-42 \%$ ) vs. $24 \%$ (95\% CI: $19-29 \%$ ), respectively, with an OR of $1.38(1.20-1.58, \mathrm{P}<0.001)$ and 1.42 (95\% CI: 1.22-1.66, $\mathrm{P}<0.001)$, respectively. However, there was no statistically significant difference in OSRly between the CRTS and SA groups; the pooled OSR1y was $71 \%$ (95\% CI: $65-78 \%$ ) vs. $68 \%$ (95\% CI: 60-76\%), respectively, and the OR was 1.06 (95\% CI: 0.94-1.19, $\mathrm{P}=0.348$ ) (Figs. 2A, 3A, 4A and 5; Table II).

Cumulative meta-analyses were performed in chronological order. With the increase in the number of cases, OR point estimates and $95 \%$ CIs of all survival rates tended to be stable and exhibited an improving trend. When multiple studies with large sample sizes were added, the effect on the outcome was only a reduction in the length of the confidence interval, reflecting an increase in the accuracy of the estimated overall treatment response. Under the $\alpha=0.05$ test standard, cumulative meta-analyses demonstrated there was no statistical difference between CRTS and SA in terms of OSRly (Fig. 2B), and the $\mathrm{P}$-value decreased gradually, stabilizing at $\mathrm{P}=0.334$ (calculated via Microsoft Excel). As regards OSR3y (Fig. 3B), it was observed that the difference was initially confirmed to be statistically significant $(\mathrm{OR}=2.10,95 \% \mathrm{CI}: 1.18-3.72, \mathrm{P}<0.05)$ when adding a 113 sample size study by Walsh et al (10) in 1996 under the selection criteria. The P-value was $>0.05$ when subsequent studies were added successively and the analysis was re-accumulated, and it again became $<0.05$ when including a 100 sample size study by Urba et al (12) in 2001 (OR=1.45, 95\% CI: $1.04-2.02, \mathrm{P}<0.05)$. Subsequently, the cumulative analysis of successively included studies demonstrated that the difference was statistically significant, with P-values stable at $<0.05$. As regards OSR5y (Fig. 4B), cumulative meta-analyses demonstrated that the difference was initially statistically significant in 2007, when a 102 sample size study was conducted by Cao et al (18) $(\mathrm{OR}=1.33,95 \%$ CI: 1.06-1.66, $\mathrm{P}<0.05)$, after which time the $\mathrm{P}$-values were stable at $<0.05$.

Surgical factors. The CRTS group had a significantly higher $\mathrm{R} 0$ resection rate and a lower local recurrence and distant 
A

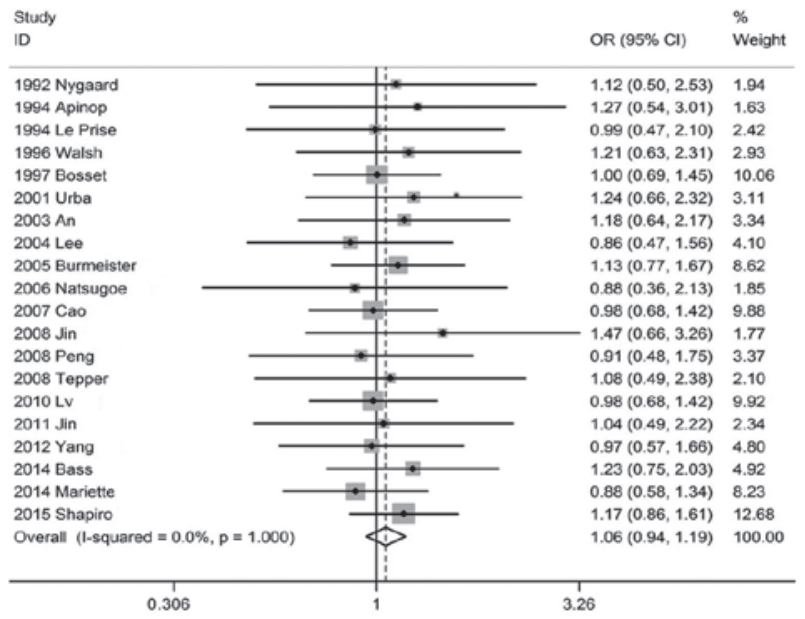

B

Study

ID

1992 Nygaard

1994 Apinop

1994 Le Prise

1996 Walsh

1997 Bosset

2001 Urba

2003 An

2004 Lee

2005 Burmeister

2006 Natsugoe

2007 Cao

$2008 \mathrm{Jin}$

2008 Peng

2008 Tepper

2010 Lv

2011 Jin

2012 Yang

2014 Bass

2014 Mariette

2015 Shapiro
OR $(95 \% \mathrm{Cl})$

$1.12(0.50,2.53)$

$1.19(0.66,2.15$

$1.11(0.70,1.77)$

$1.14(0.78,1.67)$
$1.07(0.82,1.39)$

$1.07(0.82,1.39)$
$1.09(0.86,1.39)$

$1.10(0.88,1.38)$

$1.07(0.87,1.32)$

$1.08(0.90,1.30)$

$1.07(0.90,1.29)$

$1.06(0.90,1.24)$

$1.06(0.91,1.24)$

$1.06(0.91,1.24)$

$1.05(0.91,1.21)$

$1.05(0.91,1.21)$
$1.05(0.91,1.21)$

$1.05(0.91,1.21)$
$1.04(0.91,1.19)$

$1.06(0.93,1.20)$

$1.06(0.92,1.18)$

$1.06(0.94,1.19)$

Figure 2. Traditional and cumulative meta-analysis for 1-year overall survival rate. OR, odds ratio; CI, confidence interval.

A

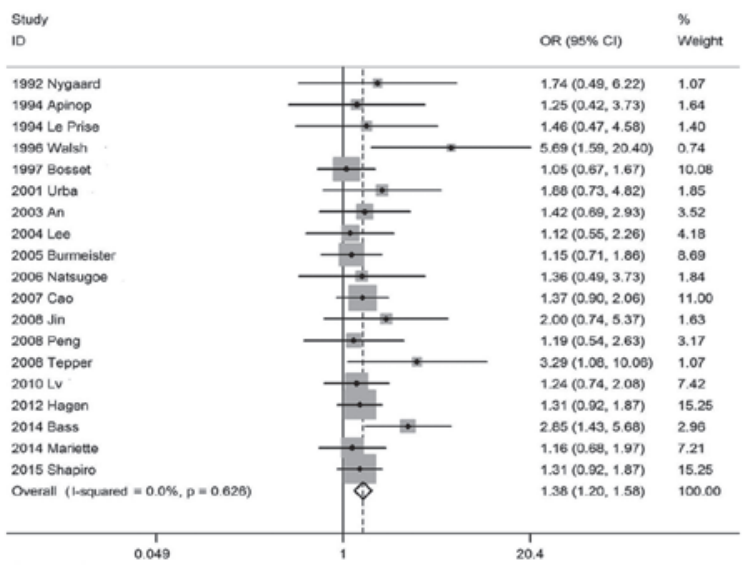

B

\begin{tabular}{ll|ll} 
Study \\
ID
\end{tabular}

Figure 3. Traditional and cumulative meta-analysis for 3-year overall survival rate. OR, odds ratio; CI, confidence interval.

A

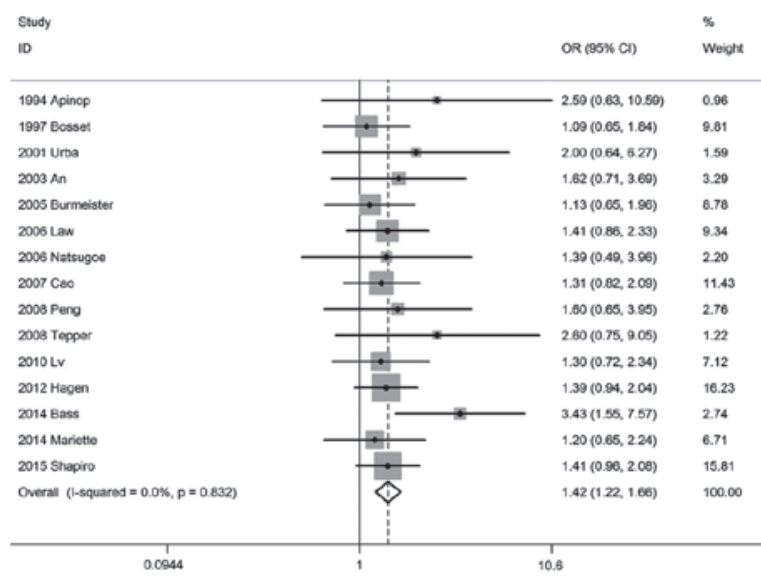

B

\begin{tabular}{|c|c|c|}
\hline \multicolumn{3}{|l|}{ Study } \\
\hline \multicolumn{2}{|l|}{ ID } & OR (95\% Cl) \\
\hline 1994 Apinop & 1 & $2.59(0.63,10.59)$ \\
\hline 1997 Bosset & 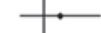 & $1.22(0.75,1.99)$ \\
\hline 2001 Urba & 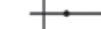 & $1.32(0.85,2.07)$ \\
\hline $2003 \mathrm{An}$ & $\rightarrow$ & $1.39(0.94,2.05)$ \\
\hline 2005 Burmeister & $\rightarrow$ & $1.29(0.94,1.78)$ \\
\hline 2006 Law & $\cdot-$ & $1.33(1.01,1.74)$ \\
\hline 2006 Natsugoe & $\because$ & $1.33(1.03,1.73)$ \\
\hline 2007 Cao & $\rightarrow$ & $1.33(1.06,1.66)$ \\
\hline 2008 Peng & $\rightarrow$ & $1.34(1.08,1.67)$ \\
\hline 2008 Tepper & $\rightarrow-$ & $1.37(1.10 .1 .70)$ \\
\hline $2010 \mathrm{LV}$ & $\rightarrow$ & $1.36(1.11,1.67)$ \\
\hline 2012 Hagen & $\rightarrow$ & $1.37(1.14,1.64)$ \\
\hline 2014 Bass & $\rightarrow$ & $1.44(1.21,1.71)$ \\
\hline 2014 Mariette & $\rightarrow$ & $1.42(1.20,1.68)$ \\
\hline 2015 Shapiro & $\rightarrow$ & $1.42(1.22,1.66)$ \\
\hline
\end{tabular}

Figure 4. Traditional and cumulative meta-analysis for 5-year overall survival rate. OR, odds ratio; CI, confidence interval.

metastasis rate compared with the $\mathrm{SA}$ group, with a pooled $\mathrm{OR}$ of 2.76 (95\% CI: 2.15-3.53, P<0.001), 0.49 (95\% CI: 0.36-6.65,
$\mathrm{P}<0.001)$ and 0.76 (95\% CI: 0.60-0.97, $\mathrm{P}=0.02)$, respectively; the differences were statistically significant. However, the 
Table II. Survival rate and surgical parameters of patients with EC by treatment approach.

\begin{tabular}{lccccc}
\hline & & \multicolumn{2}{c}{ No. of patients } & & \\
Variables & No. of studies & CRTS & SA & OR (95\% CI) & P-value \\
\hline Survival rate & 20 & 1,424 & 1,429 & $1.06(0.94-1.19)$ & 0.348 \\
OSR1y & 19 & 1,479 & 1,488 & $1.38(1.20-1.58)$ & $<0.0001$ \\
OSR3y & 15 & 1,361 & 1,437 & $1.42(1.22-1.66)$ & $<0.0001$ \\
OSR5y & & & & & \\
Surgery conditions & 9 & 774 & 874 & $2.76(2.15-3.53)$ & $<0.0001$ \\
R0 resection rate & 10 & 668 & 679 & $0.49(0.36-0.65)$ & $<0.0001$ \\
Local recurrence rate & 10 & 668 & 679 & $0.76(0.60-0.97)$ & 0.02 \\
Distant metastasis rate & 15 & 1,086 & 1,205 & $0.97(0.72-1.32)$ & 0.87 \\
Postoperative mortality & 15 & & & & \\
\hline
\end{tabular}

The fixed-effects model was used. EC, esophageal carcinoma; CRTS, neoadjuvant chemoradiotherapy followed by surgery; SA, surgery alone; OR, odds ratio; CI, confidence interval; OSR, overall survival rate; y, year.

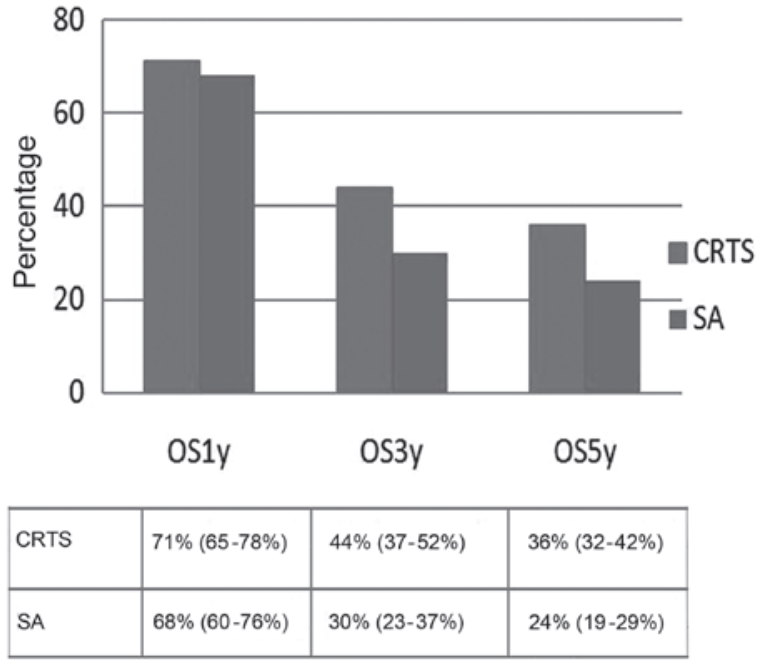

Figure 5. Survival rate and (95\% confidence interval) for CRTS and SA. OS1y, 1-year overall survival; OS3y, 3-year overall survival; OS5y, 5-year overall survival; CRTS, neoadjuvant chemoradiotherapy and surgery; SA, surgery alone.

incidence of postoperative mortality in the two groups suggested there was no significantly statistical difference, with an OR of 0.97 (95\% CI: 0.72-1.32, P=0.87) (Fig. 6, Table II).

\section{Subgroup analysis}

Survival rate of squamous cell carcinoma and adenocarcinoma. The pooled OR of squamous cell carcinoma in terms of OSR3y and OSR5y in the CRTS and SA groups was 1.57 (95\% CI: 1.21-2.04, $\mathrm{P}=0.0006)$ and 1.69 (95\% CI: 1.32-2.16, $\mathrm{P}<0.0001)$, respectively; the differences were statistically significant. However, there was no statistically significant difference in terms of OSR1y $(\mathrm{OR}=1.13,95 \% \mathrm{CI}$ : 0.88-1.45, $\mathrm{P}=0.35$ ). Compared with adenocarcinoma patients treated with SA, the OSR1y, OSR3y and OSR5y were significantly higher in CRTS, with an OR of 1.55 (95\% CI: 1.09-2.20, $\mathrm{P}=0.01), 1.77$ (95\% CI: 1.34-2.36, $\mathrm{P}<0.0001)$ and $1.92(95 \%$
CI: $1.34-2.75, \mathrm{P}=0.0004)$, respectively; the differences were statistically significant (Table III).

Survival rates of different countries or regions. The subgroup analysis of OSR3y, OSR5y for Asian, European and American populations were significantly higher in the CRTS group compared with those in the SA group, and the differences were all statistically significant $(\mathrm{P}<0.05)$. However, when comparing the OSR1y between the two groups in patients from the three continents, the difference was not significant (P>0.05; Table III).

Publication bias. A funnel plot analysis of all the studies was performed in the meta-analysis of OSR1y, OSR2y and OSR $3 y$ between CRTS and SA. This indicated that the publication bias was low in the present meta-analysis (Fig. 7).

\section{Discussion}

CRT is quickly becoming the neoadjuvant treatment of choice for patients with resectable esophageal carcinoma prior to surgery. However, trials and meta-analyses on this subject are limited and varied, with small sample sizes and heterogeneity of population distribution characteristics, tumor pathological types, tumor location, radiation doses, chemotherapy regimens, surgical approach, postoperative care and adequacy of surgical resections, despite all the advantages of trimodality therapy.

In the CROSS trial (26), CRTS improved the long-term overall and progression-free survival in patients with resectable esophageal carcinoma; this improvement was statistically significant and clinically relevant for both the adenocarcinoma and squamous cell carcinoma subtypes. In addition, locoregional control and distant disease control also improved significantly. However, Mariette et al (25) reported that, compared with SA, CRTS with cisplatin plus fluorouracil did not improve R0 resection rate or survival, but rather enhanced postoperative mortality in patients with resectable esophageal carcinoma. Burmeister et al (15) obtained results 


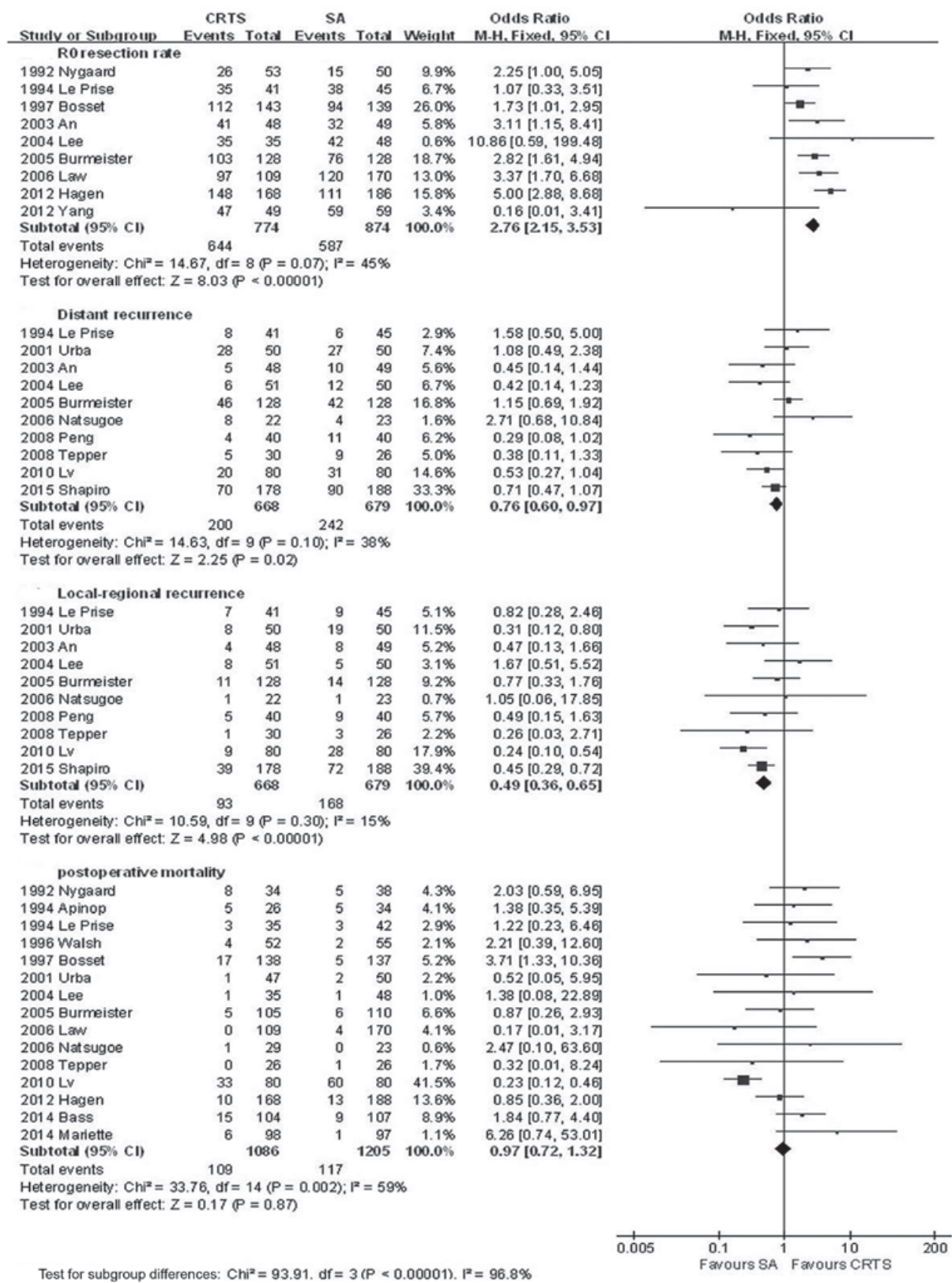

Figure 6. Traditional meta-analysis for surgical parameters of patients with EC by treatment schedule (CRTS or SA). CRTS, neoadjuvant chemoradiotherapy and surgery; SA, surgery alone; CI, confidence interval.

in a randomised controlled phase III trial indicating that preoperative CRT with cisplatin and fluorouracil did not significantly improve progression-free or overall survival in patients with resectable esophageal cancer compared with SA.

Meta-analyses on CRTS vs. SA in esophageal cancer, however, are discordant. In the most recent meta-analysis of 13 studies on CRTS compared with SA in operable patients, the hazard ratio for all-cause mortality was $0.78(\mathrm{P}<0.001)$, favoring CRTS. However, due to the large majority of locally advanced cases included in the trials and the heterogeneity in staging methods, there was no definitive conclusion regarding survival benefit for stage I or II esophageal cancer (29). A meta-analysis of those trials by Gluud and Krag (30) reported a short-term survival benefit for neoadjuvant chemoradiotherapy over surgical monotherapy in adenocarcinoma as well as squamous cell carcinoma of the esophagus. In addition, a meta-analysis by Huang et al (31) reported that CRTS with paclitaxel plus platinum appeared to be a better choice compared with platinum plus 5-fluorouracil for esophageal cancer, particularly for squamous cell carcinoma. Wijnhoven et al (32) performed a secondary meta-analysis of six published meta-analyses to compare the differences in the studies included and statistical methods applied, and found heterogeneity between the RCTs included in the meta-analyses with regard to the previously mentioned content. Of note, the majority of RCTs were conducted in the 90s; hence, the diagnostic methods, staging, treatment delivery and outcome assessment reflected the clinical practice during tha decade. 
Table III. Survival rate by histological type and continent in EC patients treated with CRTS and SA.

\begin{tabular}{|c|c|c|c|c|c|c|}
\hline \multirow[b]{2}{*}{ Variables } & \multirow[b]{2}{*}{ Overall survival } & \multirow[b]{2}{*}{ No. of studies } & \multicolumn{2}{|c|}{ No. of patients } & \multirow[b]{2}{*}{ OR (95\% CI) } & \multirow[b]{2}{*}{ P-value } \\
\hline & & & CRTS & SA & & \\
\hline \multicolumn{7}{|c|}{ Histological type } \\
\hline \multirow[t]{3}{*}{$\mathrm{SCC}$} & OSR1y & 11 & 647 & 654 & $1.13(0.88-1.45)$ & 0.35 \\
\hline & OSR3y & 10 & 554 & 556 & $1.57(1.21-2.04)$ & 0.0006 \\
\hline & OSR5y & 8 & 622 & 698 & $1.69(1.32-2.16)$ & $<0.0001$ \\
\hline \multirow[t]{3}{*}{$\mathrm{AC}$} & OSR1y & 4 & 295 & 302 & $1.55(1.09-2.20)$ & 0.01 \\
\hline & OSR3y & 5 & 429 & 442 & $1.77(1.34-2.36)$ & $<0.0001$ \\
\hline & OSR5y & 4 & 371 & 387 & $1.92(1.34-2.75)$ & 0.0004 \\
\hline \multicolumn{7}{|l|}{ Location } \\
\hline \multirow[t]{3}{*}{ Asia } & OSR1y & 8 & 398 & 403 & $1.05(0.74-1.49)$ & 0.80 \\
\hline & OSR3y & 8 & 424 & 424 & $1.81(1.37-2.40)$ & $<0.0001$ \\
\hline & OSR5y & 7 & 452 & 514 & $1.73(1.31-2.27)$ & $<0.0001$ \\
\hline \multirow[t]{3}{*}{ Europe } & OSR1y & 7 & 669 & 673 & $1.22(0.96-1.54)$ & 0.10 \\
\hline & OSR3y & 8 & 847 & 860 & $1.74(1.42-2.14)$ & $<0.0001$ \\
\hline & OSR5y & 5 & 701 & 719 & $1.69(1.35-2.13)$ & $<0.0001$ \\
\hline \multirow[t]{3}{*}{ USA } & OSR1y & 2 & 80 & 76 & $1.75(0.86-3.55)$ & 0.06 \\
\hline & OSR3y & 2 & 80 & 76 & $3.55(1.68-7.49)$ & 0.0009 \\
\hline & OSR5y & 2 & 80 & 76 & $2.80(1.19-6.61)$ & 0.02 \\
\hline
\end{tabular}

The fixed-effects model was used. EC, esophageal carcinoma; CRTS, neoadjuvant chemoradiotherapy and surgery; SA, surgery alone; OR, odds ratio; CI, confidence interval; OSR, overall survival rate; y, year; SCC, squamous cell carcinoma; AC, adenocarcinoma.

Our aim was to conduct a meta-analysis combining the traditional and cumulative methods. The traditional meta-analysis revealed that CRTS may improve the long-term survival and surgical parameters, and reduce locoregional cancer recurrence and distant metastasis in adenocarcinoma as well as squamous cell carcinoma of the oesophagus, but there was no significant difference in terms of short-term survival. We focused more on the integration of various researches chronologically by using the cumulative meta-analysis. Clinical trials on a particular research topic constitute an increasing, open and continuous entity over time. Baum et al (33) first proposed the concept of cumulative meta-analysis that was first applied to clinical practice by Lau et al (34) on the basis of the traditional meta-analysis, adding studies sequentially and performing multiple meta-analyses in a sequential manner based on the time of publication, the size of the sample and the quality score of the study; whenever a new study is published, the meta-analysis may be again continued. Unlike traditional meta-analyses, which are performed only at a certain point in time, cumulative meta-analysis was performed at each time point in order to capture the variation tendency of the combined total effect, which may enable greater use of information, contribute to early detection of coherent interventions, and facilitate new research.

From forest plots of cumulative meta-analysis (performed in chronological order), it was observed that, as the number of cases increased, the test efficacy increased and the 95\% CI gradually decreased; under the $\alpha=0.05$ test standard, cumulative meta-analyses demonstrated there was no statistical difference between CRTS and SA in terms of OSR1y, and the P-value

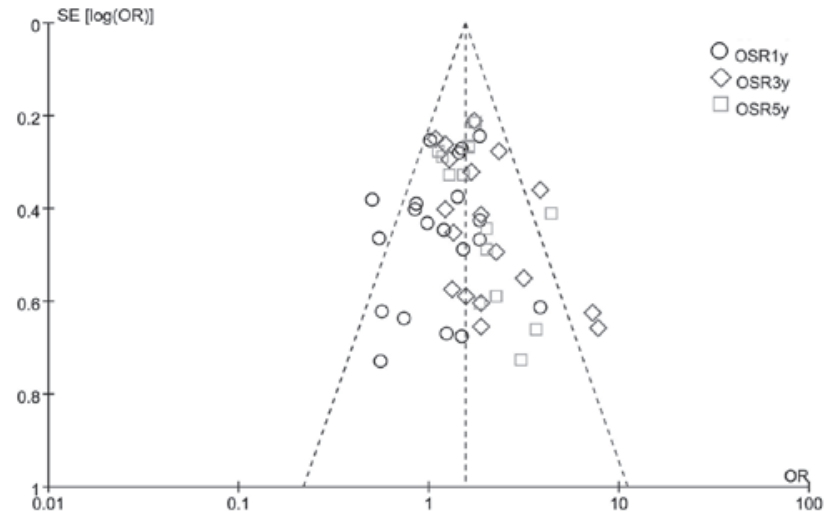

Figure 7. Funnel plot for the publication bias tests. OSR1y, 1-year overall survival rate; OSR3y, 3-year overall survival rate; OSR5y, 5-year overall survival rate; OR, odds ratio; $\mathrm{SE}$, standard error.

decreased gradually and stabilized at 0.334 . Therefore, it was concluded that CRTS did not improve the short-term survival benefit of patients with esophageal cancer. The difference between the two treatment approaches in terms of OSR3y was initially confirmed to be statistically significant $(\mathrm{OR}=2.10$, 95\% CI: 1.18-3.72, $\mathrm{P}<0.05)$; when adding a 113 sample size study by Walsh et al (10) under the selected test criteria, it was observed that the treatment regimen was the same as that of previous studies, except that the subjects were adenocarcinoma patients rather than squamous cell carcinoma patients. Thus, it was hypothesized that CRTS may be more effective in treating esophageal adenocarcinoma. The same conclusion was reached using the traditional meta-analysis, as the OR for OSR1y, OSR 3y 
and OSR5y in adenocarcinoma patients is higher compared with that in squamous cell carcinoma patients (1.55 vs. 1.13 for OSR1y, 1.77 vs. 1.57 for OSR3y and 1.92 vs. 1.69 for OSR5y). A meta-analysis conducted by Hai-Lin et al (35) also confirmed that CRTS may increase the survival rate of patients with esophageal adenocarcinoma. However, the P-value was $>0.05$ when adding a 282 sample size study by Bosset et al (11) in 1997, possibly due to the cisplatin monotherapy. The P-value again became $<0.05$ when a 100 sample size study by Urba et al (12) in 2001 was included $(\mathrm{OR}=1.45,95 \% \mathrm{CI}: 1.04-2.02, \mathrm{P}<0.05)$, in which innovative triple therapy was used, combining vinblastine with cisplatin and fluorouracil. Liu et al (36) also reported in 2015 that cisplatin with vinorelbine may achieve a higher pathological complete response rate and better survival outcomes compared with cisplatin and fluorouracil in esophageal squamous cell carcinoma. Subsequently, the cumulative analysis of successively included studies demonstrated that the difference was statistically significant, with P-values stable at $<0.05$. It was demonstrated that CRTS may improve the 3-year survival benefit of patients with esophageal cancer. As regards OSR5y, cumulative meta-analyses demonstrated that the difference was initially found to be statistically significant in 2007, when a 102 sample size study was conducted by Cao et al (17) (OR=1.33, 95\% CI: 1.06-1.66, $\mathrm{P}<0.05)$, after which the P-values were stable at $<0.05$. A study by Wolf et al (37) on long-term outcome of mitomycin $\mathrm{C}$ and 5-fluorouracil-based primary CRT for esophageal cancer demonstrated a significant increase of overall survival $(\mathrm{P}<0.0001)$ in the CRT vs. the radiotherapy alone group, indicating that CRTS may provide a long-term survival benefit to patients with esophageal cancer. However, it remains uncertain whether the alteration in the abovementioned treatment options is the cause of $\mathrm{P}<0.05$, as this is only a monistic interpretation. From the present analysis, it was concluded that CRTS was able improve the long-term survival of patients with esophageal cancer, and may be more effective in treating esophageal adenocarcinoma. In addition, vinblastine or mitomycin combined with general chemotherapy were more likely to improve the long-term survival rate following complete resection, which may also be a future research focus.

Traditional meta-analysis may be associated with various types of bias, such as selection, implementation, exit and measurement bias; the same biases may occur at various time points in the cumulative meta-analysis and affect the determination of the overall effect trend. Furthermore, certain information could not be collected (e.g., the chronological cumulative effect of the treatment regimen, the difference in efficacy and the quality score of a single article), which is a major drawback. In addition, patients included in the present study were in various stages of the trial, such as adjuvant therapy; patient compliance was also different, which may affect the results. Furthermore, the 22 included studies differed significantly in sample size; thus, the contribution to the overall effect was not proportional, which was another limitation of the cumulative meta-analysis.

In summary, it may be concluded from the cumulative meta-analysis that CRTS may increase OSR3y and OSR5y by $38 \%(\mathrm{P}<0.0001)$ and $42 \%(\mathrm{P}<0.0001)$, respectively. From the forest plot, it was observed that the difference in OSR $3 y$ and OSR5y was statistically significant, with P-values stable at $<0.05$, indicating that CRTS may improve the patient survival rate. Therefore, it is recommended that the CRTS regimen is routinely used for patients with early resectable esophageal cancer. There are ongoing studies on this subject and, as the results of those studies are published, it may further elucidate the role of CRTS in the treatment of early resectable esophageal cancer.

\section{References}

1. Ferlay J, Shin HR, Bray F, Forman D, Mathers C and Parkin DM: Estimates of worldwide burden of cancer in 2008: GLOBOCAN 2008. Int J Cancer 127: 2893-2917, 2010.

2. Parkin DM, Bray FI and Devesa SS: Cancer burden in the year 2000. The global picture. Eur J Cancer 37 (Suppl 8): S4-S66, 2001.

3. Siegel RL, Miller KD and Jemal A: Cancer statistics, 2016. CA Cancer J Clin 66: 7-30, 2016.

4. Devesa SS, Blot WJ and Fraumeni JF Jr: Changing patterns in the incidence of esophageal and gastric carcinoma in the United States. Cancer 83: 2049-2053, 1998.

5. Song Y, Li L, Ou Y, Gao Z, Li E, Li X, Zhang W, Wang J, Xu L, Zhou Y, et al: Identification of genomic alterations in oesophageal squamous cell cancer. Nature 509: 91-95, 2014.

6. Funk EM and Witte J: Multimodal therapy for esophageal adenocarcinoma. N Engl J Med 336: 375-376, author reply 375-376, 1997.

7. Nygaard K, Hagen S, Hansen HS, Hatlevoll R, Hultborn R, Jakobsen A, Mäntyla M, Modig H, Munck-Wikland E, Rosengren B, et al: Pre-operative radiotherapy prolongs survival in operable esophageal carcinoma: A randomized, multicenter study of pre-operative radiotherapy and chemotherapy. The second Scandinavian trial in esophageal cancer. World J Surg 16: 1104-1109, discussion 1110, 1992.

8. Apinop C, Puttisak $\mathrm{P}$ and Preecha N: A prospective study of combined therapy in esophageal cancer. Hepatogastroenterology 41: 391-393, 1994.

9. Le Prise E, Etienne PL, Meunier B, Maddern G, Ben Hassel M, Gedouin D, Boutin D, Campion JP and Launois B: A randomized study of chemotherapy, radiation therapy, and surgery versus surgery for localized squamous cell carcinoma of the esophagus. Cancer 73: 1779-1784, 1994.

10. Walsh TN, Noonan N, Hollywood D, Kelly A, Keeling N and Hennessy TP: A comparison of multimodal therapy and surgery for esophageal adenocarcinoma. N Engl J Med 335: 462-467, 1996.

11. Bosset JF, Gignoux M, Triboulet JP, Tiret E, Mantion G, Elias D, Lozach P, Ollier JC, Pavy JJ, Mercier M, et al: Chemoradiotherapy followed by surgery compared with surgery alone in squamouscell cancer of the esophagus. N Engl J Med 337: 161-167, 1997.

12. Urba SG, Orringer MB, Turrisi A, Iannettoni M, Forastiere A and Strawderman M: Randomized trial of preoperative chemoradiation versus surgery alone in patients with locoregional esophageal carcinoma. J Clin Oncol 19: 305-313, 2001.

13. An FS, Huang JQ, Xie YT, Chen SH and Rong TH: A prospective study of combined chemoradiotherapy followed by surgery in the treatment of esophageal carcinoma. Zhonghua Zhong Liu Za Zhi 25: 376-379, 2003 (In Chinese).

14. Lee JL, Park SI, Kim SB, Jung HY, Lee GH, Kim JH, Song HY, Cho KJ, Kim WK, Lee JS, et al: A single institutional phase III trial of preoperative chemotherapy with hyperfractionation radiotherapy plus surgery versus surgery alone for resectable esophageal squamous cell carcinoma. Ann Oncol 15: 947-954, 2004.

15. Burmeister BH, Smithers BM, Gebski V, Fitzgerald L, Simes RJ, Devitt P, Ackland S, Gotley DC, Joseph D, Millar J, et al; TransTasman Radiation Oncology Group; Australasian Gastro-Intestinal Trials Group: Surgery alone versus chemoradiotherapy followed by surgery for resectable cancer of the oesophagus: A randomised controlled phase III trial. Lancet Oncol 6: 659-668, 2005.

16. Natsugoe S, Okumura H, Matsumoto M, Uchikado Y, Setoyama T, Yokomakura N, Ishigami S, Owaki T and Aikou T: Randomized controlled study on preoperative chemoradiotherapy followed by surgery versus surgery alone for esophageal squamous cell cancer in a single institution. Dis Esophagus 19: 468-472, 2006.

17. Cao XF, He XT, Ji L, Xiao J and Lv J: Effects of neoadjuvant radiochemotherapy on pathological staging and prognosis for locally advanced esophageal squamous cell carcinoma. Dis Esophagus 22: 477-481, 2009.

18. Jin MG, Jiang SC and Chen ZW: Clinical trial of preoperative concurrent chemoradiation followed by surgery versus surgery alone for advanced esophageal carcinoma. China J Cancer Prev Treat 15: 1815-1817, 2008. 
19. Peng L, Xie TP, Han YT, et al: Randomized controlled study on preoperative concurrent chemoradiotherapy versus surgery alone for esophageal squamous cell carcinoma. Tumor Chin 28: 620-622, 2008

20. Tepper J, Krasna MJ, Niedzwiecki D, Hollis D, Reed CE, Goldberg R, Kiel K, Willett C, Sugarbaker D and Mayer R: Phase III trial of trimodality therapy with cisplatin, fluorouracil, radiotherapy, and surgery compared with surgery alone for esophageal cancer: CALGB 9781. J Clin Oncol 26: 1086-1092, 2008.

21. Lv J, Cao XF, Zhu B, Ji L, Tao L and Wang DD: Long-term efficacy of perioperative chemoradiotherapy on esophageal squamous cell carcinoma. World J Gastroenterol 16: 1649-1654, 2010.

22. Jin FL, Zu-Liang HU and Hai-Feng MA: Treatment effect of neoadjuvant chemoradiotherapy followed by surgery versus surgery alone in local advanced esophageal carcinoma. Shiyong Zhongliu Zazhi 26: 523-526, 2011.

23. Yang H, Jh F U, Liu M Z, et al: A multi-centered randomized controlled study of neo-adjuvant chemoradiotherapy followed by surgery versus surgery alone for locally advanced squamous cel carcinoma of esophagus: an interim analysis). Natl Med J Chin (Chin) 92; 1028-1032, 2006 (In Chinese).

24. Bass GA, Furlong H, O'Sullivan KE, Hennessy TP and Walsh TN: Chemoradiotherapy, with adjuvant surgery for local control, confers a durable survival advantage in adenocarcinoma and squamous cell carcinoma of the oesophagus. Eur J Cancer 50: 1065-1075, 2014

25. Mariette C, Dahan L, Mornex F, Maillard E, Thomas PA, Meunier B, Boige V, Pezet D, Robb WB, Le Brun-Ly V, et al: Surgery alone versus chemoradiotherapy followed by surgery for stage I and II esophageal cancer: Final analysis of randomized controlled phase III trial FFCD 9901. J Clin Oncol 32: 24162422,2014

26. Shapiro J, van Lanschot JJB, Hulshof MCCM, van Hagen $P$, van Berge Henegouwen MI, Wijnhoven BPL, van Laarhoven HWM, Nieuwenhuijzen GAP, Hospers GAP, Bonenkamp JJ, et al; CROSS study group: Neoadjuvant chemoradiotherapy plus surgery versus surgery alone for oesophageal or junctional cancer (CROSS): Long-term results of a randomised controlled trial. Lancet Oncol 16: 1090-1098, 2015.

27. van Hagen P, Hulshof MC, van Lanschot JJ, Steyerberg EW, van Berge Henegouwen MI, Wijnhoven BP, Richel DJ, Nieuwenhuijzen GA, Hospers GA, Bonenkamp JJ, et al; CROSS Group: Preoperative chemoradiotherapy for esophageal or junctional cancer. N Engl J Med 366: 2074-2084, 2012.

28. Law S, Kwong DL, Wong KH, Kwok KF and Wong J: The effects of neoadjuvant chemoradiation on pTNM staging and its prognostic significance in esophageal cancer. J Gastrointest Surg 10: 1301-1311, 2006
29. Sjoquist KM, Burmeister BH, Smithers BM, Zalcberg JR, Simes RJ, Barbour A and Gebski V; Australasian GastroIntestinal Trials Group: Survival after neoadjuvant chemotherapy or chemoradiotherapy for resectable oesophageal carcinoma: An updated meta-analysis. Lancet Oncol 12: 681-692, 2011.

30. Gluud LL and Krag A: Banding ligation versus beta-blockers for primary prevention in oesophageal varices in adults. Cochrane Database Syst Rev 8: CD004544, 2012.

31. Huang TC, Hsu CH, Lin CC and Tu YK: Systematic review and network meta-analysis: Neoadjuvant chemoradiotherapy for locoregional esophageal cancer. Jpn J Clin Oncol 45: 1023-1028, 2015.

32. Wijnhoven BP, van Lanschot JJ, Tilanus HW, Steyerberg EW and van der Gaast A: Neoadjuvant chemoradiotherapy for esophageal cancer: A review of meta-analyses. World J Surg 33: 2606-2614, 2009.

33. Baum ML, Anish DS, Chalmers TC, Sacks HS, Smith H Jr and Fagerstrom RM: A survey of clinical trials of antibiotic prophylaxis in colon surgery: Evidence against further use of no-treatment controls. N Engl J Med 305: 795-799, 1981.

34. Lau J, Antman EM, Jimenez-Silva J, Kupelnick B, Mosteller F and Chalmers TC: Cumulative meta-analysis of therapeutic trials for myocardial infarction. N Engl J Med 327: 248-254, 1992.

35. Jin HL, Zhu H, Ling TS, Zhang HJ and Shi RH: Neoadjuvant chemoradiotherapy for resectable esophageal carcinoma: A meta-analysis. World J Gastroenterol 15: 5983-5991, 2009.

36. Liu SL, Yang H, Zhang P, Zhang L, Zhao L, Luo LL, Fu JH, Liu MZ and Xi M: Neoadjuvant chemoradiotherapy with cisplatin plus vinorelbine versus cisplatin plus fluorouracil for esophageal squamous cell carcinoma: A matched case-control study. Radiother Oncol 116: 262-268, 2015.

37. Wolf M, Zehentmayr F, Niyazi M, Ganswindt U, Haimerl W, Schmidt M, Hölzel D and Belka C: Long-term outcome of mitomycin C- and 5-FU-based primary radiochemotherapy for esophageal cancer. Strahlenther Onkol 186: 374-381, 2010. 